\title{
Uso institucional de Twitter para combatir la infodemia causada por la crisis sanitaria de la Covid-19
}

\section{Institutional use of Twitter to combat the infodemic caused by the Covid-19 health crisis}

\author{
Marta Pulido-Polo; Víctor Hernández-Santaolalla; Ana-Alicia Lozano-González
}

Cómo citar este artículo:

Pulido-Polo, Marta; Hernández-Santaolalla, Víctor; Lozano-González, Ana-Alicia (2021). "Uso institucional de Twitter para combatir la infodemia causada por la crisis sanitaria de la Covid-19". Profesional de la información, v. 30, n. 1, e300119.

https://doi.org/10.3145/epi.2021.ene.19

Artículo recibido el 03-11-2020 Aceptación definitiva: 28-01-2021

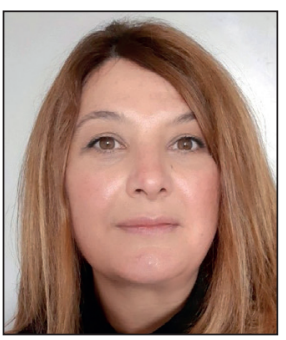

Marta Pulido-Polo $\bowtie$

https://orcid.org/0000-0001-5504-0438

Universidad de Sevilla

Facultad de Comunicación

Av. Américo Vespucio, $\mathrm{s} / \mathrm{n}$.

41092 Sevilla, España

martapulido@us.es

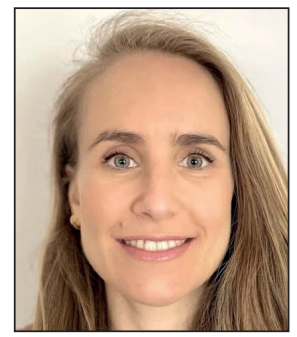

\section{Ana-Alicia Lozano-González}

https://orcid.org/0000-0002-0725-5511

Centro Universitario San Isidoro

Universidad Pablo de Olavide

Leonardo Da Vinci, 17-B.

41092 Sevilla, España

alozano@centrosanisidoro.es

\section{Resumen}

La crisis sanitaria internacional provocada por la Covid-19, más grave que las provocadas por el SARS, el MERS, la Gripe A y el Ébola, supone un desafío sin precedentes para las instituciones de todo el mundo. El objetivo principal de este trabajo es analizar el comportamiento institucional a través de Twitter para determinar si es posible inferir un modelo eficaz de comunicación institucional de crisis online de aplicación en contextos más amplios. En este sentido, se establece un diseño metodológico cuantitativo, sustentado en el análisis de contenido sobre un corpus de 995 mensajes emitidos durante el primer estado de alarma por las cuentas oficiales de Twitter de las instituciones oficiales al cargo de la gestión de la crisis sanitaria de la Covid-19 en España: @sanidadgob (483 tweets), @mitmagob (154 tweets), @ defensagob (263 tweets) e @interiorgob (95 tweets). Los resultados muestran un predominio de mensajes de empatía y seguridad (60,40\%); una distribución estable de tweets por día, concentrándose el 88,74\% de los mismos entre las 10:00 y las 20:59 horas; un uso comedido de recursos audiovisuales $(32,26 \%)$ con un enfoque eminentemente informativo $(96,18 \%)$; diferencias significativas según el estadístico chi-cuadrado con respecto al formato $\left(\chi_{(12)}^{2}=606,066 ; p<0,001\right)$ y el enfoque $\left(\chi_{(3)}^{2}=36,084 ; p<0,001\right)$ en función de la cuenta analizada, y una destacada tasa de engagement adscrita al Ministerio de Sanidad (68,96\%). Estos resultados evidencian que Twitter permite aplicar un modelo de comunicación institucional online, de fácil transferencia al contexto internacional, que sugiere una estrategia de relaciones públicas sustentada en la transparencia informativa y el goteo informativo constante.

\section{Palabras clave}

Infodemia; Contexto internacional; Comunicación institucional; Gobierno de España; Twitter; Gestión de crisis; Covid-19; Coronavirus; SARS-CoV-2; Relaciones públicas; Engagement; Confianza; Transparencia informativa. 


\begin{abstract}
The international health crisis caused by Covid-19, more serious than those caused by SARS, MERS, influenza A, and Ebola, poses an unprecedented challenge for all institutions around the world in combating the infodemic. The main objective of this work is to analyze institutional behavior through Twitter to determine whether it is possible to deduce an effective institutional online crisis communication model that is applicable in wider contexts. In this sense, a quantitative methodological design is established based on content analysis performed on a simple of 995 tweets from the official Twitter accounts of institutions in charge of managing the health crisis during the first state of alarm in Spain: @sanidadgob (483 tweets), @mitmagob (154 tweets), @defensagob (263 tweets), and @interiorgob (95 tweets). The results illustrate a predominance of empathetic and security-related messages (60.40\%); a stable distribution of tweets per day, with $88.74 \%$ of them published between $10: 00$ and $20: 59$; a moderate use of audiovisual resources (32.26\%) with a very informative approach (96.18\%); a few significant differences according to the chi-squared statistic with respect to the format $\left(\chi_{(12)}^{2}=606.066 ; p<0.001\right)$ and approach $\left(\chi_{(3)}^{2}=36.084 ; p<0.001\right)$ depending on the accounts analyzed; and a substantial level of engagement with the Spanish Ministry of Health's account (68.96\%). These results demonstrate that Twitter allows the application of an online institutional communication model that is easily transferable to an international context, suggesting a public relations strategy based on information transparency and constant information flow.
\end{abstract}

\title{
Keywords
}

Infodemic; International context; Institutional communication; Government of Spain; Twitter; Crisis management; Covid-19; Coronavirus; SARS-CoV-2; Public relations; Engagement; Trust; Information transparency.

\section{Introducción}

La crisis sanitaria generada por la Covid-19, más compleja, amplia y grave que las anteriores, pone de relieve el compromiso público y la capacidad de respuesta gubernamental a nivel internacional (Liao et al., 2020), así como la responsabilidad de las instituciones de todo el mundo de gestionar adecuadamente la comunicación con su ciudadanía desde

"la transparencia, la coherencia, el entendimiento con los distintos stakeholders, el liderazgo y la divulgación" (Costa-Sánchez; López-García, 2020, p. 11).

Estamos ante una situación sin precedentes que rompe con los modelos de gestión comunicativa de las crisis (Xifra, 2020) y en la que resulta esencial la intervención de las partes implicadas para aprovechar el poder de las redes sociales con el objetivo de divulgar información fiable y poner freno a la alarmante propagación de desinformación médica y contenido no verificable que se ha difundido sobre la Covid-19 (Kouzy et al., 2020).

Tal y como establece la Organización Mundial de la Salud, en adelante OMS, si algo hemos aprendido después de los brotes de SARS, MERS, Gripe A (H1N1) y Ébola es que la comunicación de crisis y de compromiso con la comunidad (CCCC) ${ }^{1}$ es fundamental para el éxito de este tipo de emergencias sanitarias, ya que la Covid-19

"desafía los sistemas de salud pública y su capacidad para comunicarse eficazmente con su población. La falta de comunicación lleva a la pérdida de confianza y reputación, impacto económico y, en el peor de los casos, la pérdida de vidas" (World Health Organization, 2020, p. 1).

En este sentido, el 19 de marzo de 2020, la OMS realizó un llamamiento a los líderes mundiales para garantizar que la gestión institucional de la comunicación de crisis fuera un componente esencial en la preparación y respuesta a esta emergencia sanitaria y, además de proporcionar pautas concretas de actuación (tabla 1), advirtió a los gobiernos sobre la importancia de gestionar la CCCC para:

- salvar vidas y minimizar las consecuencias adversas;

- ayudar a prevenir la infodemia y generar confianza en la respuesta institucional;

- aliviar la confusión y evitar malentendidos;

- garantizar el derecho de la población a estar informada y a comprender los riesgos a los que está expuesta;

- minimizar las divergencias acerca de la percepción del riesgo;

- adaptar conocimientos científicos complejos haciéndolos accesibles a la población para que los entiendan y confíen en ellos;

- involucrar a las comunidades en el control de los brotes y garantizar que tomen medidas de protección;

- vigilar y notificar casos, rastrear contactos, cuidar enfermos, prestar atención clínica y proporcionar apoyo a las necesidades logísticas que se requieran, y

- minimizar la disrupción social y proteger no sólo la salud, sino también el empleo, el turismo y la economía.

Desde el primer momento de la pandemia, el comportamiento comunicacional del Gobierno de España ha sido activo, ofreciendo datos actualizados a través de todo tipo de soportes y aplicaciones para hacer llegar la información a los ciudadanos, practicando la transparencia y una gestión pública positiva. Y es que

"la estrategia del discurso institucional seguida por el Gobierno persigue establecer un canal directo con la ciudadanía, interesada en recibir información sobre la situación y conocer los diversos escenarios de la actualidad" (Castillo-Esparcia; Fernández-Souto; Puentes-Rivera, 2020, p. 3). 
Es aquí donde Twitter se revela como un eficaz instrumento de gestión de la comunicación institucional (Caldevilla-Domínguez; Rodríguez-Terceño; Barrientos-Báez, 2019; Rodríguez-Fidalgo; Ruiz-Paz; Paíno-Ambrosio, 2019; Gong; Lane, 2020), especialmente durante el Estado de alarma para el Gobierno de España, y donde radica el interés de nuestro estudio.
La crisis sanitaria internacional provocada por la Covid-19 es un desafío sin precedentes para las instituciones de todo el mundo

El éxito institucional de Twitter, a nivel nacional e internacional, se sustenta en su capacidad universal de generar con la ciudadanía un sistema de comunicación dialógico (Sáez-Martín; Haro-de-Rosario; Caba-Pérez, 2015) eficaz, directo y ágil (Pérez-Gabaldón; Nicasio-Varea, 2019; Segura-Mariño; Paniagua-Rojano; Fernández-Sande, 2020). Se convierte así en un excepcional canal informativo gubernamental para compartir y consumir información relevante (en tiempo real) en situaciones de crisis provocadas por desastres y emergencias (Akar; Muraki, 2011; Burton et al., 2012; Chatfield; Scholl; Brajawidagda, 2013; Spence et al., 2015; Lachlan et al., 2019; Pourebrahim et al., 2019; Edo-Osagie et al., 2020) y combatir la infodemia.

Tabla 1. Etapas de actuación en la gestión de CCCC para países con transmisión de la Covid-19 en curso

\begin{tabular}{|c|c|}
\hline Etapa & Acciones \\
\hline $\begin{array}{l}\text { 1. Sistemas de comuni- } \\
\text { cación de riesgos }\end{array}$ & $\begin{array}{l}\text { Fortalecer la capacidad de respuesta de comunicadores y expertos en participación comunitaria. } \\
\text { Desarrollar, actualizar continuamente y compartir estrategias de comunicación de crisis y de compromiso con la } \\
\text { comunidad (CCCC) atendiendo a las necesidades de respuesta requeridas. } \\
\text { Monitorear las campañas de CCCC. }\end{array}$ \\
\hline $\begin{array}{l}\text { 2. Coordinación interna } \\
\text { y con agentes colabo- } \\
\text { radores }\end{array}$ & $\begin{array}{l}\text { Fortalecer el compromiso con los agentes colaboradores para: } \\
\text { - Compartir información de forma continua para evitar desvíos incoherentes y potencialmente conflictivos. } \\
\text { - Diversificar canales relevantes para la difusión de mensajes importantes relativos a la salud. } \\
\text { - Obtener nuevas audiencias mediante la elaboración de materiales de comunicación. } \\
\text { - Beneficiarse de los recursos humanos y financieros de terceros. } \\
\text { - Publicar materiales de manera conjunta según corresponda (por ejemplo, comunicados de prensa, informes de } \\
\text { situación, guías de protección de la salud). } \\
\text { - Ampliar el alcance de las actividades de participación comunitaria utilizando las fortalezas y oportunidades que } \\
\text { ofrecen los colaboradores. }\end{array}$ \\
\hline $\begin{array}{l}\text { 3. Comunicación } \\
\text { pública }\end{array}$ & $\begin{array}{l}\text { Identificar portavoces en función de su credibilidad ante la población, el tipo de mensaje que se debe transmitir y la } \\
\text { gravedad de la situación. } \\
\text { Asegurarse de que los mensajes sean coherentes en todos los sectores y niveles. } \\
\text { Compartir información con regularidad (lo ideal sería todos los días a la misma hora). } \\
\text { Hacer partícipe a la población del liderazgo y la toma de decisiones con argumentos claros. } \\
\text { Compartir historias, fotos y vídeos que ilustren mensajes clave. } \\
\text { Asegurarse de que el público sepa dónde obtener información actualizada con regularidad (por ejemplo, sitios web, } \\
\text { prensa, sesiones informativas, etc.). } \\
\text { Proporcionar una comunicación regular y transparente a través de los canales utilizados por nuestras audiencias. } \\
\text { Utilizar medios tradicionales, internet y redes sociales, líneas directas y mensajes de texto según corresponda. }\end{array}$ \\
\hline $\begin{array}{l}\text { 4. Compromiso con la } \\
\text { comunidad }\end{array}$ & $\begin{array}{l}\text { Mantener una comunicación bidireccional con las audiencias afectadas para comprender y responder a sus preocu- } \\
\text { paciones, actitudes, creencias y barreras para seguir las directrices sanitarias a través de mecanismos como: } \\
\text { - Líneas directas operadas por estudiantes de medicina, que pueden responder llamadas y participar en las redes } \\
\text { sociales, y } \\
\text { - Programas de radio en los que se proporciona información y el público puede hacer preguntas. } \\
\text { Realizar un seguimiento de las personas afectadas para asegurarse de que sigan las recomendaciones sanitarias e } \\
\text { identificar las barreras para que se comprometan con las medidas de protección. } \\
\text { Interactuar con influencers, principalmente sanitarios, para comunicarse con las poblaciones afectadas, especialmen- } \\
\text { te los que son difíciles de alcanzar. } \\
\text { Establecer un feedback consistente entre la población y el equipo de emergencias sanitarias, para adecuar de forma } \\
\text { efectiva sus repuestas a las necesidades concretas de protección sanitaria que requiere la población. }\end{array}$ \\
\hline $\begin{array}{l}\text { 5. Abordar la incerti- } \\
\text { dumbre y la percep- } \\
\text { ción y gestión de la } \\
\text { desinformación }\end{array}$ & $\begin{array}{l}\text { Establecer un feedback periódico y capturar preguntas, malentendidos y desinformación comunes a través de líneas } \\
\text { directas, sanitarios y la población. } \\
\text { Asegurarse de que los resultados del seguimiento de las redes sociales y los medios tradicionales se evalúen rápida- } \\
\text { mente a través del equipo específicamente creado para este propósito. } \\
\text { Interactuar con influencers para captar las percepciones de las personas a través de sus comentarios. } \\
\text { Preparar una guía de acuerdo con las percepciones y preocupaciones de la población, y difundirla en diferentes } \\
\text { canales de información. }\end{array}$ \\
\hline $\begin{array}{l}\text { 6. Creación de } \\
\text { competencias }\end{array}$ & $\begin{array}{l}\text { Asegurarse de que se comparte con el personal de respuesta de emergencias sanitarias una terminología y mensajes } \\
\text { clave claros. } \\
\text { Actualizar las competencias del personal de respuesta de CCCC a medida que se implementan nuevas metodologías } \\
\text { y campañas. } \\
\text { Considerar la formación de líderes, personal de respuesta y portavoces sobre la evolución de la CCCC según sea } \\
\text { necesario. }\end{array}$ \\
\hline
\end{tabular}

Fuente: Elaborado a partir de World Health Organization (2020, p. 4-5). 


\section{La infodemia y Twitter}

La Covid-19 ha ocasionado grandes desafíos relacionados con la gestión y la difusión de la información (Aleixandre-Benavent; Castelló-Cogollos; Valderrama-Zurián, 2020): a la avalancha de (des)información, se ha unido un incremento ingente en el consumo de información por parte de la población (Casero-Ripollés, 2020). Sólo entre el 14 y el 19 de marzo se publicó el triple de noticias que a principios de marzo (Lázaro-Rodríguez; Herrera-Viedma, 2020). De hecho, las publicaciones surgidas en los últimos meses revelan el interés académico internacional del fenómeno de la infodemia causada por la Covid-19 (Mavragani, 2020a; Higgins et al., 2020; Cousins et al., 2020; Hernández-García; Giménez-Júlvez, 2020; Park; Park; Chong, 2020; Rovetta; Bhagavathula, 2020; Solomon et al., 2020).

Aunque es la OMS quien devuelve a la actualidad el término infodemia (pandemia informativa), en referencia al exceso de información no verificada en internet y redes sociales sobre la Covid-19, el primer estudio sobre epidemiología de la (des)información en materia de salud pública en la Red es el publicado en 1996 por Davison (Eysenbach, 2002). Y, con posterioridad, ha sido ampliamente tratado en la bibliografía académica desde una perspectiva clínica (Eysenbach, 2006; 2009; 2020; Chan; Ho; Lam, 2013; Mahoney et al., 2015; Radin; Sciascia, 2017; Mavragani; Ochoa, 2019; Mavragani, 2020b; Schillinger; Chittamuru; Ramírez, 2020). En concreto, las implicaciones de Twitter con la infodemia han sido revisadas por Velardi et al. (2014); Zeraatkar y Ahmadi (2018) y Liu, Chen y Kuo (2019), destacando especialmente el estudio de Chew y Eysenbach (2010) sobre la incidencia que tuvo Twitter durante la pandemia de Gripe A.

En este contexto, durante la crisis sanitaria provocada por la Covid-19, Twitter está jugando un papel decisivo que pivota entre su capacidad para favorecer la desinformación y la difusión de información médica relevante (Rosenberg; Syed; Rezaie, 2020). Si es adecuadamente gestionada, esta red social puede convertirse en una importante herramienta institucional para la lucha contra la desinformación dada su capacidad para conectar a las instituciones oficiales con los ciudadanos (y viceversa) de manera rápida y directa (McGravey, 2020). De hecho, Twitter ha permitido a las instituciones publicar "refutaciones específicas sobre los mitos más recurrentes y perjudiciales" en torno a la crisis sanitaria provocada por la Covid-19 (Aleixandre-Benavent; Castelló-Cogollos; Valderrama-Zurián, 2020).

\section{El 19 de marzo de 2020, la OMS realizó un llamamiento a los líderes mundiales para dar respuesta a la emergencia sani- taria de la Covid desde la gestión institu- cional de la comunicación de crisis}

\section{Comunicación institucional, relaciones públicas y Twitter}

Las redes sociales ofrecen a las organizaciones un canal único para construir y mantener relaciones interactivas fluidas (Wigand, 2010) con sus públicos (Killian; McManus, 2015; McCann; Barlow, 2015), permitiendo gestionar las interrelaciones entre las instituciones públicas y la sociedad (Yi; Oh; Kim, 2013), tal y como se propugna desde la perspectiva relacional de las relaciones públicas (Ledingham; Brunning, 1998; 1999; Ledingham, 2001; 2003).

En el contexto actual, la comunicación es eminentemente digital (Pătruț, 2015) y viene determinada no sólo por el contenido y los usuarios, sino también por las tecnologías existentes (Keïnanen; Kuivalainen, 2015). De esta forma, si bien internet ha contribuido a potenciar el uso de la comunicación online (Jayarama; Manraib; Manraib, 2015), las redes sociales se han convertido en plataformas de comunicación estratégica (Guesalaga, 2016) que han creado interesantes desafíos para los profesionales de la comunicación organizacional (Evans; Twomey; Talan, 2011).

Twitter se afianza como la red social más utilizada por gobiernos e instituciones de todo el mundo en sus estrategias de comunicación digital (Khan; Yoon; Park, 2014; Panagiotopoulos; Bigdeli; Sams, 2014; Leone; Delli-Paoli; Senatore, 2015; Meijer; Torenvlied, 2016; Park et al., 2016; Waisbord; Amado, 2017; Castillo-Esparcia; Castillero-Ostio; Castillo-Díaz, 2020), puesto que se posiciona como una red de movilización social, con la que se consigue una gran difusión que permite llegar a la mayoría del público (Arroyo-Almaraz; Calle-Mendoza; Van-Wyk, 2018). Debido a su inmediatez, horizontalidad y sencillez en la publicación y difusión de contenidos, ha supuesto un cambio en la forma en la que los tres actores de la comunicación política (actores públicos, mediáticos y ciudadanos) interactúan entre sí (Suau-Gomila et al., 2017). Este cambio se ha producido en todos los ámbitos comunicativos, incluso en los de gran impacto social como es la comunicación en situaciones de crisis y emergencia: en los orígenes de la pandemia, las redes sociales fueron el medio en el que se transmitió una información que podría haber salvado muchas vidas (Aleixandre-Benavent; Castelló-Cogollos; Valderrama-Zurián, 2020).

\section{Objetivos}

El Real decreto 463/2020, de 14 de marzo (España, 2020), por el que se declara el Estado de alarma para la gestión de la situación de crisis sanitaria ocasionada por la Covid-19, establece en su artículo 4. "Autoridad competente", que, bajo la superior dirección de la Presidencia del Gobierno, las autoridades competentes delegadas (en sus respectivas áreas de responsabilidad) al frente de la crisis sanitaria provocada por la Covid-19 durante el Estado de alarma son: el Ministerio de Defensa, el Ministerio del Interior, el Ministerio de Transportes, Movilidad y Agenda Urbana y el Ministerio de Sanidad. En este sentido, el objetivo principal de este trabajo es analizar el comportamiento comunicacional que estas instituciones, implicadas directamente en la gestión de la crisis sanitaria que nos ocupa, han llevado a cabo a través de Twitter, para determinar su adecuación a las recomendaciones de gestión mundial de la comunicación de crisis y de 
compromiso con la comunidad (CCCC) y observar si es posible inferir un modelo eficaz de comunicación institucional de crisis online específico mediante esta red social de aplicación funcional en el contexto internacional.

Para alcanzar el objetivo principal se establecen los siguientes objetivos específicos:

OE1: Determinar cuáles son los diferentes contenidos temáticos difundidos durante el estado de alarma atendiendo a los mensajes clave en situaciones de crisis.

OE2: Observar la regularidad de la información compartida atendiendo a la periodicidad de las publicaciones por día y hora.

OE3: Concretar cuáles son, en su caso, los recursos adicionales que se incluyen en cada tweet para reforzar el mensaje clave.

OE4: Identificar el volumen de interacciones de los mensajes en términos de número de comentarios, "me gusta", retweets y reproducciones de los vídeos contenidos para, posteriormente, determinar la tasa de engagement.

La consecución de los objetivos anteriores permitiría analizar el grado de adecuación del comportamiento comunicacional observado con los indicadores de gestión institucional de crisis relativos, principalmente, a la comunicación pública (OE1, OE2 y OE3, en relación con las especificaciones de la OMS en este punto acerca del tipo de mensaje, su regularidad y los recursos empleados), compromiso de la comunidad (OE1, OE2 y OE4, en relación con las especificaciones sobre compromiso, temática y regularidad) y gestión de la desinformación (OE1, OE2 y OE4, en relación con el feedback, la regularidad y el contenido) para así intentar conocer hasta qué punto Twitter se ha erigido como una de las aplicaciones clave para la CCCC, según los indicadores de la OMS, y si esta red puede convertirse en un medio eficaz para la comunicación institucional de crisis online desde los postulados relacionistas extrapolable a ámbitos supranacionales dadas las características universales inherentes a Twitter.

\section{Metodología}

Tomando como referencia las metodologías validadas por Fernández-Gómez, Hernández-Santaolalla y Sanz-Marcos (2018); Tuñón-Navarro, Bouza-García y Carral (2019); Chen, Lerman y Ferrara (2020), y Ruiz-del-Olmo y Bustos-Díaz (2020), entre otros, relativas al análisis de Twitter desde una perspectiva comunicacional, para alcanzar los objetivos de esta investigación se establece un diseño metodológico de corte cuantitativo. En concreto, se aplica un análisis de contenido sobre un corpus de 995 mensajes emitidos durante la vigencia del Estado de alarma, entre el 14 de marzo (fecha en la que entra en vigor por la publicación del Real decreto 463/2020 en el BOE) y el 21 de junio (fecha en que finaliza tras las sucesivas prórrogas aprobadas en el Congreso de los Diputados), por las cuenta oficiales de Twitter de las instituciones oficiales al cargo de la gestión de la crisis sanitaria de la Covid-19 en España:

- @sanidadgob: 483 tweets;

- @mitmagob: 154 tweets;

- @defensagob: 263 tweets;

- @interiorgob: 95 tweets.

Para la localización de los tweets se utilizó el sistema de búsqueda avanzada de Twitter, analizándose finalmente todos los mensajes emitidos por las cuatro cuentas anteriores que contenían el hashtag oficial \#EsteVirusLoParamosUnidos, eje de la campaña institucional puesta en marcha por el Gobierno de España.

La codificación fue realizada entre el 15 y el 30 de julio de 2020 por tres jueces, quienes tras el correspondiente período de entrenamiento alcanzaron un índice de acuerdo intercodificadores de 0,853 según el coeficiente alfa de Krippendorff (2004). La ficha de análisis y el libro de códigos se diseñaron a partir de los trabajos de Capriotti, Zeler y Oliveira (2019) y Costa-Sánchez y López-García (2020), sustentado este último en las aportaciones de Reynolds y Seeger (2005), Vos y Buckner (2015) y Jang y Park (2018). Al respecto, se contemplaron cuatro bloques principales: contenido; regularidad de la información; recursos de refuerzo del mensaje clave, e interacción y engagement, que proporcionarían los resultados para responder, respectivamente, a cada uno de los cuatro objetivos planteados en el epígrafe anterior.

1. Contenido: se codificó según su adscripción a los niveles:

1) advertencia y preparación (precrisis: se informa a la población del riesgo, futuros eventos adversos y recomendaciones orientadas a reducir el daño);

2) empatía y seguridad (evento inicial: se traslada empatía y seguridad, fomentándose el entendimiento general de las circunstancias de la crisis y sus consecuencias);

3) entendimiento público (mantenimiento: se propicia un entendimiento público más preciso de los riesgos, se corrigen desinformaciones y se explican las acciones de respuesta);

4) recuperación y reconstrucción (resolución: se informa y persuade sobre los esfuerzos de recuperación y reconstrucción, facilitando una discusión abierta sobre la causa, la culpabilidad, la responsabilidad, la adecuación de la respuesta y los nuevos riesgos);

5) lecciones aprendidas (evaluación: se acometen mejoras específicas en la comunicación de crisis y la capacidad de respuesta);

6) otros (mensajes no clasificables en los niveles anteriores) (Costa-Sánchez; López-García, 2020, p. 4). 
2. Regularidad de la información: se identificó día y hora de publicación del tweet.

3. Recursos de refuerzo del mensaje clave: se contabilizó si el texto del tweet iba acompañado de algún otro tipo de información (imagen, vídeo, GIF, enlace a información actualizada) y si este tenía un enfoque informativo o interactivo, es decir, si se limitaba a la difusión pública de información o si estimulaba la participación a través de comentarios, acudir a otro sitio web, etc.

4. Interacción y engagement: se contabilizó el número de "me gusta", retweets, comentarios y respuestas a los comentarios desde las cuentas oficiales -lo cual ha sido analizado como indicador de interacción y conversación (Criado; Martínez-Fuentes; Silván, 2013; Ramos-Serrano; Fernández-Gómez; Pineda-Cachero, 2018)-, así como las reproducciones de los vídeos -cuando el mensaje lo contenía-. Esta información permitía, a su vez, obtener la tasa de engagement, concretada en la tasa de apoyo (total de likes entre el número de seguidores por cien), la tasa de viralización (total de retweets entre el número de seguidores por cien), la tasa de conversación (total de comentarios entre el número de seguidores por cien) y la tasa general de engagement, que es la suma de las tres anteriores (Capriotti; Zeler; Oliveira, 2019, p. 1102).

\section{Resultados}

En cuanto a la variable referida al contenido de los mensajes (tabla 2), se observa un protagonismo de la categoría empatía y seguridad (60,40\%), seguida por la de entendimiento público (25,33\%). Asimismo, si se atiende a cada una de las cuentas analizadas, se encuentran dife-

\section{Twitter está demostrando ser una herra- mienta universal clave en la lucha contra la desinformación}

rencias significativas en cuanto al contenido transmitido en función del organismo $\left(\chi_{(15)}^{2}=489,569 ; p<0,001\right)$. Al respecto, se observa que, así como el Ministerio de Transportes, Movilidad y Agenda Urbana orienta algo más de la mitad de sus mensajes al entendimiento público, mediante la explicación de los riesgos y la información de la toma de decisiones a la población, el Ministerio de Sanidad utiliza prácticamente el mismo porcentaje de tweets a transmitir una sensación de seguridad e intentar reducir la agitación emocional de la ciudadanía; un contenido/cometido que ocupa también la práctica totalidad de los mensajes del Ministerio de Defensa, como queda ilustrado en los siguientes ejemplos.

[Emoji de "Bustos en silueta"] \#MaríaJoséRallo: "Los datos de movilidad muestran el alto grado de compromiso de la ciudadanía" para acabar con el \#Covid19.

Como dice el mensaje de @Renfe: \#siemprehayluzalfinaldeltunel

\#EsteVirusLoParamosUnidos [emoji de "Bíceps flexionado"]

(@mitmagob, 29 de marzo de 2020).

Todo el personal de las Fuerzas Armadas, sea cual sea la misión encomendada y en el lugar en el que se nos requiera, sabe que el objetivo prioritario de la \#OperaciónBalmis es y será ayudar a salvar vidas.

\#EsteVirusLoParamosUnidos

(@defensagob, 28 de abril de 2020).

[Emoji de “Cámara de vídeo"] Fernando Simón, director del CCAES:

[Emoji de "Gráfico con tendencia hacia abajo"] Los datos de \#Covid19 son favorables, pero al mismo tiempo nos indican que todavía se detectan casos nuevos y hay un riesgo de transmisión y por tanto no debemos bajar la guardia.

https://mscbs.gob.es/profesionales/saludPublica/ccayes/alertasActual/nCov-China/documentos/Actualizacion_103_Covid-19.pdf

\#EsteVirusLoParamosUnidos

(@sanidadgob, 12 de mayo de 2020).

Tabla 2. Contenido de los tweets por cuenta de usuario (\%)

\begin{tabular}{|c|c|c|c|c|c|}
\hline & @sanidadgob & @mitmagob & @defensagob & @interiorgob & Total \\
\hline Advertencia y preparación & 2,07 & 0,00 & 0,00 & 0,00 & 1,01 \\
\hline Empatía y seguridad & 59,83 & 25,97 & 94,30 & 25,26 & 60,40 \\
\hline Entendimiento público & 27,33 & 59,09 & 2,28 & 24,21 & 25,33 \\
\hline Recuperación y reconstrucción & 3,31 & 0,65 & 0,00 & 3,16 & 2,01 \\
\hline Lecciones aprendidas & 5,80 & 9,09 & 0,00 & 3,16 & 4,52 \\
\hline Otros & 1,66 & 5,19 & 3,42 & 44,21 & 6,73 \\
\hline Total & 483 & 154 & 263 & 95 & 995 \\
\hline
\end{tabular}

En cuanto a la regularidad de la información, en el gráfico 1 puede observarse la distribución de los mensajes emitidos por cada una de las cuentas y el conjunto de estas a lo largo del continuo temporal, y en la tabla 3 los estadísticos descriptivos de los mensajes por usuario y día. Al respecto, se observa cómo la distribución de tweets por día se mantuvo bastante estable entre los organismos, notándose una bajada generalizada de la actividad a partir del 23 de mayo, coinci- 
diendo con el inminente inicio (el 25 de mayo) de la fase 2 de la desescalada y la progresiva mayor flexibilización de las restricciones derivadas de la emergencia sanitaria provocada por la Covid-19. Del mismo modo, se observan algunos picos de actividad que, si bien en ocasiones son bastante generalizados, como el 13 de abril o el 3 y el 4 de mayo, esta última, en la que comenzó la fase cero, en otros se limitan a cuentas determinadas. De esta forma, así como los días de mayor actividad del Ministerio de Transportes, Movilidad y Agenda Urbana y del Ministerio de Sanidad fueron precisamente los dos últimos señalados, el día de mayor actividad de la cuenta del Ministerio del Interior fue el 24 de marzo, mientras que el del Ministerio de Defensa fue el 26 de abril. En cuanto a la hora de publicación de los tweets, el $88,74 \%$ de los mismos se emitieron entre las 10:00 y las 20:59 (tabla 4). A continuación, se recogen, a modo de ejemplo, algunos tweets de los días indicados:

Y nuestro reconocimiento hoy va a todas las niñas y niños que se están portando como unos campeones en estos días difíciles [emojis de "Bebé", "Chica" y "Chico"]

Y también va a las madres y a los padres, que están haciendo un esfuerzo diario titánico [emojis de "Familia (mujer, niña, niño)" y “Familia (hombre, niña, niño)"]

¡GRACIAS!

\#EsteVirusLoParamosUnidos

(@interiorgob 24 de marzo de 2020).

\#OperaciónBalmis

En esta batalla contra el \#Covid19, los hombres y mujeres de nuestras Fuerzas Armadas están muy cerca de los más vulnerables; demostrando, una vez más, su vocación de servicio y su gran calidad humana.

\#EsteVirusLoParamosUnidos

(@defensagob, 26 de abril de 2020).

[Emojis de "Megáfono" y "Marcadores"] A partir de mañana será obligatorio el uso de mascarillas en el transporte público.

El @boegob publica hoy una \#OM donde se fijan los requisitos para garantizar una \#movilidad segura conforme al Plan de nueva normalidad.

https://bit.ly/2WfaOgl

\#EsteVirusLoParamosUnidos

(@mitmagob, 3 de mayo de 2020).

[Emoji de "Calendario"] ¿Qué implica la \#FASEO?

[Emoji de "Poste de barbero"] Se reactiva la actividad comercial y determinados servicios profesionales, siempre cumpliendo unos requisitos de aforo, distancia, las medidas de higiene y aforo.

Más información en @boegob $\downarrow$

https://boe.es/boe/dias/2020/05/03/pdfs/BOE-A-2020-4793.pdf

\#EsteVirusLoParamosUnidos

(@sanidadgob, 4 de mayo de 2020).
30

25

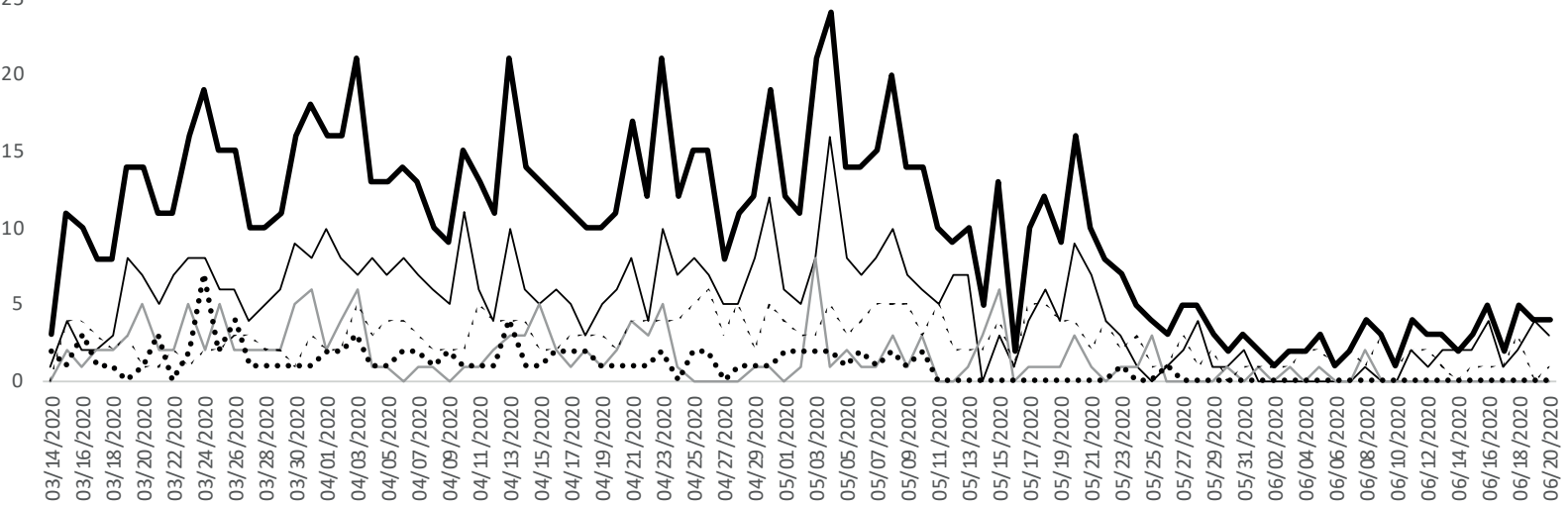

Total 
Tabla 3. Estadísticos descriptivos de tweets por día

\begin{tabular}{|c|c|c|c|c|c|}
\hline & @sanidadgob & @mitmagob & @defensagob & @interiorgob & Total \\
\hline Media & 4,88 & 1,55 & 2,66 & 0,96 & 10,04 \\
\hline Desviación típica & 3,25 & 1,72 & 1,44 & 1,15 & 5,64 \\
\hline Máximo de mensajes/día & 16 & 8 & 6 & 7 & 24 \\
\hline Mínimo de mensajes/día & 0 & 0 & 0 & 0 & 1 \\
\hline Total & 483 & 154 & 263 & 95 & 995 \\
\hline
\end{tabular}

Tabla 4. Distribución de tweets por horas y cuenta de usuario (\%)

\begin{tabular}{|c|c|c|c|c|c|}
\hline & @sanidadgob & @mitmagob & @defensagob & @interiorgob & Total \\
\hline 00:00-00:59 & 0,00 & 0,00 & 0,00 & 0,00 & 0,00 \\
\hline 01:00-01:59 & 0,00 & 0,00 & 0,00 & 0,00 & 0,00 \\
\hline 02:00-02:59 & 0,00 & 0,00 & 0,00 & 0,00 & 0,00 \\
\hline 03:00-03:59 & 0,00 & 0,00 & 0,00 & 0,00 & 0,00 \\
\hline 04:00-04:59 & 0,00 & 0,00 & 0,00 & 0,00 & 0,00 \\
\hline 05:00-05:59 & 0,00 & 0,00 & 0,00 & 0,00 & 0,00 \\
\hline 06:00-06:59 & 0,41 & 0,00 & 0,00 & 0,00 & 0,20 \\
\hline 07:00-07:59 & 0,21 & 0,00 & 0,00 & 0,00 & 0,10 \\
\hline 08:00-08:59 & 1,24 & 0,00 & 0,00 & 0,00 & 0,60 \\
\hline 09:00-09:59 & 5,59 & 0,00 & 4,18 & 7,37 & 4,52 \\
\hline 10:00-10:59 & 5,59 & 3,25 & 10,65 & 4,21 & 6,43 \\
\hline 11:00-11:59 & 12,84 & 11,69 & 11,79 & 4,21 & 11,56 \\
\hline $12: 00-12: 59$ & 6,63 & 12,99 & 11,41 & 3,16 & 8,54 \\
\hline 13:00-13:59 & 9,52 & 7,79 & 10,65 & 15,79 & 10,15 \\
\hline $14: 00-14: 59$ & 8,07 & 11,69 & 7,60 & 24,21 & 10,05 \\
\hline $15: 00-15: 59$ & 6,42 & 5,19 & 1,90 & 6,32 & 5,03 \\
\hline 16:00-16:59 & 6,21 & 1,95 & 4,18 & 7,37 & 5,13 \\
\hline 17:00-17:59 & 7,66 & 7,14 & 4,56 & 4,21 & 6,43 \\
\hline 18:00-18:59 & 5,59 & 12,99 & 6,84 & 4,21 & 6,93 \\
\hline 19:00-19:59 & 9,52 & 8,44 & 6,84 & 6,32 & 8,34 \\
\hline 20:00-20:59 & 8,90 & 9,09 & 14,45 & 6,32 & 10,15 \\
\hline 21:00-21:59 & 4,14 & 6,49 & 3,42 & 4,21 & 4,32 \\
\hline $22: 00-22: 59$ & 1,45 & 1,30 & 1,52 & 2,11 & 1,51 \\
\hline 23:00-23:59 & 0,00 & 0,00 & 0,00 & 0,00 & 0,00 \\
\hline Total & 483 & 154 & 263 & 95 & 995 \\
\hline
\end{tabular}

Con respecto al formato de los mensajes, como puede observarse en la tabla 5, existe una disparidad en función de la cuenta analizada, aunque en general las opciones de vídeo o imagen suelen ser las mayoritarias. Asimismo, en cuanto al enfoque, si bien priman los mensajes de carácter informativo -ilustrados en los primeros dos tweets apuntados a continuación-, también pueden encontrarse algunos mensajes de índole interactiva, especialmente en el caso del Ministerio de Transportes, Movilidad y Agenda Urbana -como ejemplifica el tercero de los tweets siguientes-, y en menor medida en el perfil del Ministerio de Sanidad. Al respecto, destaca la cuenta del Ministerio de Defensa, donde todos los mensajes buscan la mera transmisión de información. En cualquier caso, se observan diferencias significativas según el estadístico chi-cuadrado con respecto al formato $\left(\chi_{(12)}^{2}=606,066 ; p<0,001\right)$ y el enfoque $\left(\chi_{(3)}^{2}=36,084 ; p<0,001\right)$ en función de la cuenta analizada.

Desde la puesta en marcha de la \#OperaciónBalmis, el @EjercitoAire ha realizado 34 aerotransportes, 14 de ellos vuelos internacionales, con material sanitario y equipamiento de protección.

\#EsteVirusLoParamosUnidos

(@defensagob, 26 de abril de 2020).

Publicados los datos actualizados de \#Covid2019

Consulta aquí la situación en España https://covid19.isciii.es

\#EsteVirusLoParamosUnidos

(@sanidadgob, 26 de marzo de 2020). 
[...] Ante el brote \#coronavirus, es importante disponer de información contrastada y confirmada.

[...]Suscríbete a nuestro canal oficial de \#Telegram para estar al día de todas las actualizaciones.

[...] Súmate aquí: https://t.me/mitmagob

\#EsteVirusLoParamosUnidos

\#QuédateEnCasa [...]

(@mitmagob, 20 de marzo de 2020).

Tabla 5. Formato y enfoque de los tweets por cuenta de usuario (\%)

\begin{tabular}{|l|c|c|c|c|c|c|}
\cline { 2 - 6 } \multicolumn{1}{l|}{} & @sanidadgob & @ @itmagob & @defensagob & @interiorgob & Total \\
\hline Formato & 6,63 & 18,83 & 84,03 & 14,74 & 29,75 \\
\hline Imagen & 45,34 & 31,17 & 12,93 & 21,05 & 32,26 \\
\hline Vídeo & 4,97 & 11,04 & 0,76 & 0,00 & 4,32 \\
\hline GIF & 20,29 & 29,22 & 1,52 & 17,89 & 16,48 \\
\hline Otros & 22,77 & 9,74 & 0,76 & 46,32 & 17,19 \\
\hline Link a información & \multicolumn{7}{|l|}{} \\
\hline Enfoque & 95,65 & 89,61 & 100,00 & 98,95 & 96,18 \\
\hline Informativo & 4,35 & 10,39 & 0,00 & 1,05 & 3,82 \\
\hline Interactivo & 483 & 154 & 263 & 95 & 995 \\
\hline Total & \multicolumn{7}{|l|}{} \\
\hline
\end{tabular}

Por último, con respecto a las interacciones del resto de los usuarios con los mensajes emitidos por los organismos oficiales, en la tabla 6 se observan, en general, buenos niveles si se atiende a la media de los valores, destacando a la baja la cuenta del Ministerio de Transportes, Movilidad y Agenda Urbana, que, a su vez, es la que cuenta con un menor número de seguidores. Sin embargo, los altos valores de las desviaciones típicas indican que existe una gran variación en cuanto a los "me gusta", retweets y comentarios alcanzados por cada mensaje. Así, por ejemplo, el 72,9\% de los mensajes emitidos por la cuenta del Ministerio de Sanidad cuenta con menos de 400 likes, mientras que, por el margen superior, sólo seis tweets alcanzan la cifra de 2.000, habiendo un salto de más de 10.000 "me gusta" entre el primero y el segundo.

Por otro lado, resulta interesante el bajo índice de respuestas que las cuentas oficiales ofrecen a los comentarios de los usuarios, lo que negaría la utilidad conversacional de Twitter, al menos entre institución y usuario.

Finalmente, atendiendo a las tasas de engagement, la cuenta que obtiene una tasa general más elevada es la del Ministerio de Sanidad, seguida por la del Ministerio de Defensa. Estas dos son, además, las que cuentan con una mayor tasa de

Tabla 6. Total de interacciones y tasa de engagement

\begin{tabular}{|c|c|c|c|c|c|c|}
\hline & & @sanidadgob & @mitmagob & @defensagob & @interiorgob & Total \\
\hline Seguidores & & 553.541 & 105.724 & 397.748 & 724.645 & \\
\hline Tweets & & 483 & 154 & 263 & 95 & 995 \\
\hline \multirow{3}{*}{ Me gusta } & M & 410,44 & 36,3 & 537,67 & 252,57 & 371,09 \\
\hline & SD & 842,864 & 45,839 & 810,64 & 488,141 & 753,232 \\
\hline & Total & 198.242 & 5.590 & 141.407 & 23.994 & 369.233 \\
\hline \multirow{3}{*}{ Retweets } & M & 310,46 & 29,91 & 151,50 & 137,42 & 208,50 \\
\hline & SD & 481,501 & 43,834 & 341,585 & 286,749 & 403,218 \\
\hline & Total & 149953 & 4606 & 39844 & 13055 & 207458 \\
\hline \multirow{3}{*}{ Comentarios } & M & 69,46 & 5,08 & 16,30 & 47,61 & 43,36 \\
\hline & SD & 113,83 & 9,819 & 38,953 & 63,859 & 88,53 \\
\hline & Total & 33551 & 783 & 4286 & 4523 & 43143 \\
\hline \multirow{3}{*}{$\begin{array}{l}\text { Respuestas a } \\
\text { comentarios }\end{array}$} & M & 0,13 & 0,28 & 0,03 & 0,04 & 0,12 \\
\hline & SD & 0,672 & 0,867 & 0,268 & 0,289 & 0,607 \\
\hline & Total & 64 & 43 & 7 & 4 & 118 \\
\hline \multirow{2}{*}{$\begin{array}{l}\text { Reproducción de } \\
\text { vídeo* }\end{array}$} & M & 89,06 & 3,53 & 42,04 & 27,07 & 67,36 \\
\hline & SD & 618,10 & 5,82 & 71,82 & 25,51 & 511,44 \\
\hline TdA & & 35,81 & 5,29 & 35,55 & 3,31 & \\
\hline $\mathrm{TdV}$ & & 27,09 & 4,36 & 10,02 & 1,80 & \\
\hline $\mathrm{TdC}$ & & 6,06 & 0,74 & 1,08 & 0,62 & \\
\hline TEG & & 68,96 & 10,38 & 46,65 & 5,74 & \\
\hline
\end{tabular}

* Para calcular los estadísticos descriptivos de las reproducciones de vídeo sólo se tuvieron en cuenta los mensajes que contenían alguno. 
apoyo y de viralización, siendo esta última bastante superior en el caso de la cuenta @sanidadgob, lo que significa que los tweets han sido compartidos un mayor número de veces por cada cien seguidores. También el Ministerio de Sanidad es el que cuenta con una mayor tasa de conversación, aunque esta sigue siendo muy reducida. Al cruzar dicha tasa de engagement con el resto de variables analizadas, se observa que sólo existen diferencias estadísticamente significativas entre esta y el formato $\left(\chi_{(12)}^{2}=23,031 ; p<0,03\right)$, pero no así entre la tasa de engagement y el contenido o el enfoque. En este sentido, se aprecia que son aquellos mensajes que contaban con apoyo audiovisual, especialmente vídeos, los que generaban un mayor engagement.

\section{Discusión y conclusiones}

Twitter se revela a priori como un medio clave para la transmisión regular, constante y pertinente de la información bajo el paradigma de la transparencia de las organizaciones: un vehículo ideal para construir y mantener relaciones interactivas intensivas con los públicos (Grunig, 2009) y generar así una confianza en la opinión pública (Hucker, 2020) que se manifiesta en el número de interacciones y en el engagement (Lazarus; Thornton, 2020). Es un sistema eficaz de comunicación institucional de crisis que parte de su capacidad (si es adecuadamente gestionada) de posicionar a las instituciones como fuentes informativas fiables. Es uno de los antídotos clave para luchar contra la infodemia y es fruto, fundamentalmente, de la gestión de los contenidos que se transmiten, la regularidad de la información, los recursos empleados y la bidireccionalidad implícita que se mantiene con los públicos.

Se evidencia una concentración de mensajes en torno a las medidas adoptadas frente a la crisis, la desescalada y las recomendaciones sociosanitarias. Twitter permite a las instituciones la emisión de contenidos en tiempo real, muy eficaces en situaciones de crisis severas como la que nos ocupa.

Los resultados obtenidos en este estudio se alinean con los trabajos previos de Reynolds y Seeger (2005), Vos y Buckner (2015) y Jang y Park (2018) sobre los tipos de mensajes asociados a las cinco etapas en la gestión de la comunicación en situaciones de crisis (Costa-Sánchez; López-García, 2020, p. 4).

La periodicidad de la información compartida por los citados ministerios españoles coincide tanto con las recomendaciones de Jordan (2017), que establece que lo ideal es publicar entre uno y dos posts al día, como con las franjas horarias más utilizadas para conectarse a las redes sociales según el Estudio Anual de Redes Sociales de 2019 (IAB Spain, 2019), que se concentran en torno a dos sesiones principales de mañana y tarde. Estos datos confirmarían un goteo informativo que se adapta a las necesidades informativas detectadas en la población y a sus hábitos de consumo informativo. Facilitar los mensajes cuando más pueden interesar favorece una comunicación efectiva con los públicos. Se optimiza así una asimilación de los mensajes institucionales que coadyuva, consecuentemente, a contrarrestar tímidamente la infodemia, pues si bien el volumen de información se sigue incrementando, se intenta priorizar los mensajes procedentes de fuentes oficiales. En otras palabras, si bien no habría una mejora en cuanto a cantidad -algo que sólo se conseguiría reduciendo el número de mensajes de las distintas fuentes-, sí que se atisba una intención de optimización de la calidad de la información.

Se evidencia que los recursos audiovisuales son los más utilizados para ilustrar el mensaje clave a transmitir, favoreciendo, a su vez, la generación de engagement. De hecho, al relacionar el resto de variables estudiadas y la tasa de engagement, sólo se obtuvieron relaciones estadísticamente significativas en el caso de la variable formato, resolviéndose cómo aquellos mensajes que utilizaban contenido audiovisual, especialmente vídeos, generaban un mayor compromiso por parte de los usuarios, lo cual está en consonancia con los hallazgos de Capriotti, Zeler y Oliveira (2019, p. 1098).

El engagement está determinado principalmente por el alto número de me gusta, retweets y comentarios obtenidos. Sin embargo, la interactividad real, que implicaría la respuesta de la cuenta oficial a los comentarios de los usuarios es muy reducida, lo que pondría en entredicho la capacidad conversacional de la red social (Ramos-Serrano; Fernández-Gómez; Pineda-Cachero, 2018). En cualquier caso, destacan los niveles de compromiso en la cuenta del $\mathrm{Mi}$ nisterio de Sanidad que, al fin y al cabo, es la que se podía entender como la más idónea para informar en esta situación de crisis sanitaria que estamos analizando.

Como conclusión final, se puede afirmar que el comportamiento comunicacional en Twitter de los ministerios al frente de la crisis sanitaria derivada de la Covid-19 se adscriben firmemente a cinco de las recomendaciones en materia de comunicación pública emitidas por la World Health Organization (2020) relativa a las etapas para la gestión de la CCCC. En este sentido, si bien se observa una infrautilización para la gestión del compromiso con la comunidad, el uso de Twitter por parte de los ministerios analizados permitiría abordar la incertidumbre y gestionar la desinformación entre la población. De este modo, el comportamiento comunicacional del Gobierno de España, en el período analizado, se focaliza en el posicionamiento de las autoridades delegadas al frente de la crisis sanitaria como una fuente de información pública constante y fiable, implicando así

"una clara estrategia de relaciones públicas, trabajando la imagen favorable de modo indirecto o implícito, a través de la información y la transparencia en la gestión, entendida esta como la rendición pública de cuentas" (Castillo-Esparcia; Fernández-Souto; Puentes-Rivera, 2020, p. 18). 


\section{Notas}

1. Del original inglés RCCE, acrónimo de risk communication and community engagement.

\section{Referencias}

Akar, Adam; Muraki, Yuya (2011). "Twitter for crisis communication: lessons learned from Japan's tsunami disaster". International journal of web based communities, v. 7, n. 3, pp. 392-402.

https://doi.org/10.1504/IJWBC.2011.041206

Aleixandre-Benavent, Rafael; Castelló-Cogollos, Lourdes; Valderrama-Zurián, Juan-Carlos (2020). "Información y comunicación durante los primeros meses de Covid-19. Infodemia, desinformación y papel de los profesionales de la información". El profesional de la información, v. 29, n. 4, e290408.

https://doi.org/10.3145/epi.2020.jul.08

Arroyo-Almaraz, Isidoro; Calle-Mendoza, Samuel; Van-Wyk, Cliff (2018). "La eficacia en la comunicación de las ONGD. El uso de Facebook en campañas de emergencia". Revista latina de comunicación social, n. 73, pp. 765-789.

https://doi.org/10.4185/RLCS-2018-1281

Burton, Scott H.; Tanner, Kesler W.; Giraud-Carrier, Christophe G.; West, Joshua H.; Barnes, Michael D. (2012). “'Right time, right place'. Health communication on Twitter: value and accuracy of location information". Journal of medical internet research, v. 14, n. 6, e156. https://doi.org/10.2196/jmir.2121

Caldevilla-Domínguez, David; Rodríguez-Terceño, José; Barrientos-Báez, Almudena (2019). "El malestar social a través de las nuevas tecnologías: Twitter como herramienta política". Revista latina de comunicación social, n. 74, pp. 1264-1290. https://doi.org/10.4185/RLCS-2019-1383

Capriotti, Paul; Zeler, lleana; Oliveira, Andrea (2019). “Comunicación dialógica 2.0 en Facebook. Análisis de la interacción en las organizaciones de América Latina". Revista latina de comunicación social, n. 74, pp. 1094-1113.

https://doi.org/10.4185/RLCS-2019-1373

Casero-Ripollés, Andreu (2020). "Impact of Covid-19 on the media system. Communicative and democratic consequences of news consumption during the outbreak". El profesional de la información, v. 29, n. 2, e290223.

https://doi.org/10.3145/epi.2020.mar.23

Castillo-Esparcia, Antonio; Castillero-Ostio, Elisabeth; Castillo-Díaz, Ana (2020). "Los think tanks en España. Análisis de sus estrategias de comunicación digitales". Revista latina de comunicación social, n. 77, pp. 253-273.

https://www.doi.org/10.4185/RLCS-2020-1457

Castillo-Esparcia, Antonio; Fernández-Souto, Ana-Belén; Puentes-Rivera, Iván (2020). "Comunicación política y Covid-19. Estrategias del Gobierno de España". El profesional de la información, v. 29, n. 4, e290419.

https://doi.org/10.3145/epi.2020.jul.19

Chan, Kit-Lam; Ho, Soo-Yong; Lam, Thai-Hing (2013). "Infodemiology of alcohol use in Hong Kong mentioned on blogs: infoveillance study". Journal of medical internet research, v. 15, n. 9, e192.

https://doi.org/10.2196/jmir.2180

Chatfield, Akemi-Takeoka; Scholl, Hans-Jochen; Brajawidagda, Uuf (2013). "Tsunami early warnings via Twitter in government: Net-savvy citizens' co-production of time-critical public information services". Government information quarterly, v. 30, n. 4, pp. 377-386.

https://doi.org/10.1016/j.giq.2013.05.021

Chen, Emily; Lerman, Kristina; Ferrara, Emilio (2020). "Tracking social media discourse about the Covid-19 pandemic: development of a public coronavirus Twitter data set. JMIR public health and surveillance, v. 6, n. 2, e19273. https://doi.org/10.2196/19273

Chew, Cynthia; Eysenbach, Gunther (2010). "Pandemics in the age of Twitter: content analysis of tweets during the 2009 H1N1 outbreak". PLoS one, v. 5, n. 11, e14118.

https://doi.org/10.1371/journal.pone.0014118

Costa-Sánchez, Carmen; López-García, Xosé (2020). “Comunicación y crisis del coronavirus en España. Primeras lecciones". Profesional de la información, v. 29, n. 3, e290304.

https://doi.org/10.3145/epi.2020.may.04

Cousins, Henry C.; Cousins, Clara C.; Harris, Alon; Pasquale, Louis R. (2020). "Regional infoveillance of Covid-19 case rates: analysis of search-engine query patterns". Journal of medical internet research, v. 22, n. 7, e19483.

https://doi.org/10.2196/19483

Criado, Juan-Ignacio; Martínez-Fuentes, Guadalupe; Silván, Aitor (2013). “Twitter en campaña: las elecciones municipales españolas de 2011". Revista de investigaciones políticas y sociológicas, v. 12, n. 1, pp. 93-113.

https://revistas.usc.gal/index.php/rips/article/view/1307 
Edo-Osagie, Oduwa; De-La-Iglesia, Beatriz; Lake, lain; Edeghere, Obaghe (2020). “A scoping review of the use of Twitter for public health research". Computers in biology and medicine, n. 122, e103770.

https://doi.org/10.1016/j.compbiomed.2020.103770

España (2020). "Real decreto 463/2020, de 14 de marzo, por el que se declara el estado de alarma para la gestión de la situación de crisis sanitaria ocasionada por el Covid-19". BOE, n. 67, 14 marzo.

https://www.boe.es/buscar/doc.php?id=BOE-A-2020-3692

Evans, Angelica; Twomey, Jane; Talan, Scott (2011). “Twitter as a public relations tool”. Public relations journal, v. 5, n. 1, pp. $15-31$. https://www.researchgate.net/publication/265278938_Twitter_as_a_Public_Relations_Tool

Eysenbach, Gunther (2002). "Infodemiology: the epidemiology of (mis)information". The American journal of medicine, v. 113 , n. 9, pp. 763-765.

https://doi.org/10.1016/S0002-9343(02)01473-0

Eysenbach, Gunther (2006). "Infodemiology: tracking flu-related searches on the web for syndromic surveillance". In: AMIA Annual symposium procedures, pp. 244-248.

https://www.ncbi.n/m.nih.gov/pmc/articles/PMC1839505

Eysenbach, Gunther (2009). "Infodemiology and infoveillance: framework for an emerging set of public health informatics methods to analyze search, communication and publication behavior on the internet". Journal of medical internet research, v. 11, n. 1, e11.

https://doi.org/10.2196/jmir.1157

Eysenbach, Gunther (2020). "How to fight an infodemic: the four pillars of infodemic management". Journal of medical internet research, v. 22, n. 6, e21820.

https://doi.org/10.2196/21820

Fernández-Gómez, Jorge-David; Hernández-Santaolalla, Víctor; Sanz-Marcos, Paloma (2018). “Influencers, marca personal e ideología política en Twitter". Cuadernos.info, n. 42, pp. 19-37.

https://scielo.conicyt.cl/scielo.php?script=sci_arttext\&pid=S0719-367X2018000100019

Gong, Xi; Lane, Maria D. (2020). "Institutional Twitter usage among U.S. geography departments". The professional geographer, v. 72, n. 2, pp. 219-237.

https://doi.org/10.1080/00330124.2019.1653770

Grunig, James E. (2009). “Paradigms of global public relations in an age of digitalisation”. PRism, n. 6, pp. 1-19. https://www.prismjournal.org/uploads/1/2/5/6/125661607/v6-no2-a1.pdf

Guesalaga, Rodrigo (2016). "The use of social media in sales: individual and organizational antecedents, and the role of customer engagement in social media". Industrial marketing management, v. 54, pp. 71-79.

https://doi.org/10.1016/j.indmarman.2015.12.002

Hernández-García, Ignacio; Giménez-Júlvez, Teresa (2020). "Assessment of health information about Covid-19 prevention on the internet: infodemiological study". JMIR public health and surveillance, v. 6, n. 2, e18717.

https://doi.org/10.2196/18717

Higgins, Thomas S.; Wu, Arthur W.; Sharma, Dhruv; Illing, Elisa A.; Rubel, Kolin; Ting, Jonathan Y. (2020). "Correlations of online search engine trends with Coronavirus disease (Covid-19) incidence: infodemiology study". JMIR public health and surveillance, v. 6, n. 2, e19702.

https://doi.org/10.2196/19702

Hucker, Daniel (2020). Public opinion and twentieth-century diplomacy: a global perspective. London: Bloomsbury academic. ISBN: 9781472527165

IAB Spain (2019). Estudio anual de redes sociales 2019. IAB Spain.

https://iabspain.es/wp-content/uploads/2019/06/estudio-anual-redes-sociales-iab-spain-2019_vreducida.pdf

Jang, Kyungeun; Park, Namkee (2018). "The effects of repetitive information communication through multiple channels on prevention behavior during the 2015 MERS outbreak in South Korea". Journal of health communication, v. 23, n. 7, pp. 670-678. https://doi.org/10.1080/10810730.2018.1501440

Jayarama, Dureen; Manraib, Ajay K.; Manraib, Lalita A. (2015). "Effective use of marketing technology in Eastern Europe: web analytics, social media, customer analytics, digital campaigns and mobile applications". Journal of economics, finance and administrative science, v. 20, n. 39, pp. 118-132.

https://doi.org/10.1016/j.jefas.2015.07.001

Jordan, Katy (2017). "Examining the UK higher education sector through the network of institutional accounts on Twitter". First Monday, v. 22, n. 5, pp. 1-28.

http://oro.open.ac.uk/49233/3/FirstMonday_accepted.pdf 
Keinänen, Hanna; Kuivalainen, Olli (2015). "Antecedents of social media B2B use in industrial marketing context: Customers' view". Journal of business \& industrial marketing, v. 30, n. 6, pp. 711-722.

https://doi.org/10.1108/JBIM-04-2013-0095

Khan, Gohar-Feroz; Yoon, Ho-Young; Park, Han-Woo (2014). "Social media communication strategies of government agencies: Twitter use in Korea and the USA". Asian journal of communication, v. 24, n. 1, p. 60-78.

https://doi.org/10.1080/01292986.2013.851723

Killian, Ginger; McManus, Kristy (2015). “A marketing communications approach for the digital era: managerial guidelines for social media integration". Business horizons, v. 58, n. 5, pp. 539-549.

https://doi.org/10.1016/j.bushor.2015.05.006

Kouzy, Ramez; Jaoude, Joseph-Abi; Kraitem, Afif; El-Alam, Moli B.; Karam, Basil; Zarka, Jabra; Traboulsi, Cindy; Akl, Elie W.; Baddour, Khalil (2020). "Coronavirus goes viral: quantifying the Covid-19 misinformation epidemic on Twitter". Cureus, v. 12, n. 3, e7255.

https://doi.org/10.7759/cureus.7255

Krippendorff, Klaus (2004). "Reliability in content analysis. Some common misconceptions and recommendations". Human communication research, v. 30, n. 3, pp. 411-433.

https://doi.org/10.1111/j.1468-2958.2004.tb00738.x

Lachlan, Kenneth A.; Xu, Zhan; Hutter, Emily E.; Adam, Rainear; Spence, Patric R. (2019). "A little goes a long way: serial transmission of Twitter content associated with hurricane Irma and implications for crisis communication". Journal of strategic innovation and sustainability, v. 14, n. 1, pp. 16-26.

https://cutt.ly/9kvcxcB

https://doi.org/10.33423/jsis.v14i1.984

Lázaro-Rodríguez, Pedro; Herrera-Viedma, Enrique (2020). "Noticias sobre Covid-19 y 2019-nCoV en medios de comunicación de España: el papel de los medios digitales en tiempos de confinamiento". Profesional de la información, v. 29, n. 3, e290302.

https://doi.org/10.3145/epi.2020.may.02

Lazarus, Jeffrey; Thornton, Judd R. (2020). "Bully pulpit? Twitter users' engagement with President Trump's tweets". Social science computer review, v. 31, n. 5, pp. 625-643.

https://doi.org/10.1177/0894439320946578

Ledingham, John A. (2001). "Government-community relationships: extending the relational theory of public relations". Public relations review, v. 27, n. 3, pp. 285-295.

https://doi.org/10.1016/S0363-8111(01)00087-X

Ledingham, John A. (2003). "Explicating relationship management as a general theory of public relations". Journal of public relations research, v. 15, n. 2, pp. 181-198.

https://doi.org/10.1207/S1532754XJPRR1502_4

Ledingham, John A.; Bruning, Stephen D. (1998). "Relationship management in public relations: dimensions of an organization-public relationship". Public relations review, v. 24, n. 1, pp. 55-65.

https://doi.org/10.1016/S0363-8111(98)80020-9

Ledingham, John A.; Bruning, Stephen D. (1999). "Relationships between organizations and publics: development of a multi-dimensional organization-public relationship scale". Public relations review, v. 25, n. 2, pp. 157-170. https://doi.org/10.1016/S0363-8111(99)80160-X

Leone, Stefania; Delli-Paoli, Angela; Senatore, Daniela (2015). "Social media communication in central governments: the case of Twitter activity of Italian ministries". Journal of communications research, v. 7, n. 4, 413-429.

https://cutt.ly/okvcFM9

Liao, Qiuyan; Yuan, Jiehu; Dong, Meihong; Yang, Lin; Fielding, Richard; Lam, Wendy-Wing-Tak (2020). “Public engagement and government responsiveness in the communications about Covid-19 during the early epidemic stage in China: infodemiology study on social media data". Journal of medical internet research, v. 22, n. 5, e18796.

https://doi.org/10.2196/18796

Liu, Sam; Chen, Brian; Kuo, Alex (2019). "Monitoring physical activity levels using Twitter data: infodemiology study". Journal of medical internet research, v. 21, n. 6, e12394.

https://doi.org/10.2196/12394

Mavragani, Amaryllis (2020a). "Tracking Covid-19 in Europe: infodemiology approach". JMIR Public health and surveillance, v. 6, n. 2, e18941.

https://doi.org/10.2196/18941 
Mavragani, Amaryllis (2020b). "Infodemiology and infoveillance: scoping review”. Journal of medical internet research, v. 22, n. 4, e16206. https://doi.org/10.2196/16206

Mavragani, Amaryllis; Ochoa, Gabriela (2019). "Google trends in infodemiology and infoveillance: methodology framework. JMIR Public health and surveillance, v. 5, n. 2, e13439.

https://doi.org/10.2196/13439

Mahoney, L. Meghan; Tang, Tang; Ji, Kai; Ulrich-Schad, Jessica (2015). "The digital distribution of public health news surrounding the human papillomavirus vaccination: a longitudinal infodemiology study. JMIR Public health and surveillance, v. 1, n. 1, e2. https://publichealth.jmir.org/2015/1/e2/?utm_source=TrendMD\&utm_medium=cpc\&utm_campaign=JMIR_TrendMD_0

McCann, Margaret; Barlow, Alexis (2015), "Use and measurement of social media for SMEs". Journal of small business and enterprise development, v. 22, n. 2, pp. 273-287.

https://doi.org/10.1108/JSBED-08-2012-0096

McGravey, Kevin (2020). "Digital public forums: Power and representation in the internet's public squares". New political science, v. 42, n. 3, pp. 253-271.

https://doi.org/10.1080/07393148.2020.1807274

Meijer, Albert-Jacob; Torenvlied, René (2016). "Social media and the new organization of government communications: an empirical analysis of Twitter usage by the Dutch police". The American review of public administration, v. 46, n. 2, pp. $143-161$. https://doi.org/10.1177/0275074014551381

Panagiotopoulos, Panagiotis; Bigdeli, Alinaghi-Ziaee; Sams, Steven (2014). "Citizen-government collaboration on social media: the case of Twitter in the 2011 riots in England". Government information quarterly, v. 31, n. 3, pp. $349-357$. https://doi.org/10.1016/j.giq.2013.10.014

Park, Han-Woo; Park, Sejung; Chong, Miyoung (2020). "Conversations and medical news frames on Twitter: infodemiological study on Covid-19 in South Korea". Journal of medical internet research, v. 22, n. 5, e18897.

https://doi.org/10.2196/18897

Park, Min-Jae; Kang, Dongsuk; Rho, Jae-Jeung; Lee, Duk-Hee (2016). "Policy role of social media in developing public trust: Twitter communication with government leaders. Public management review, v. 18, n. 9, pp. 1265-1288.

https://doi.org/10.1080/14719037.2015.1066418

Pătruț, Monica (2015). “Candidates in the presidential elections in Romania (2014): the use of social media in political marketing". Studies and scientific researches. Economics edition, n. 21, pp. 127-135.

https://doi.org/10.29358/sceco.v0i21.309

Pérez-Gabaldón, Marta; Nicasio-Varea, Blanca (2019). “Comunicación parlamentaria 2.0: el debate de la moción de censura en Twitter". Miguel Hernández communication journal, v. 10, n. 1, pp. 147-164.

https://dialnet.unirioja.es/servlet/articulo?codigo $=6784667$

Pourebrahim, Nastaran; Sultana, Selima; Edwards, John; Gochanour, Amanda; Mohantyc, Somya (2019). "Understanding communication dynamics on Twitter during natural disasters: a case study of Hurricane Sandy". International journal of disaster risk, v. 37, pp. 101-176.

https://doi.org/10.1016/j.ijdrr.2019.101176

Radin, Massimo; Sciascia, Savino (2017). "Infodemiology of systemic lupus erythematous using Google Trends". Lupus, v. 26, n. 8 , pp. 886-889.

https://doi.org/10.1177/0961203317691372

Ramos-Serrano, Marina; Fernández-Gómez, Jorge-David; Pineda-Cachero, Antonio (2018). "'Follow the closing of the campaign on streaming': the use of Twitter by Spanish political parties during the 2014 European elections". New media \& society, v. 20, n. 1, pp. 122-140.

https://doi.org/10.1177/1461444816660730

Reynolds, Barbara; Seeger, Matthew W. (2005). "Crisis and emergency risk communication as an integrative model". Journal of health communication, v. 10, n. 1, pp. 43-55.

https://doi.org/10.1080/10810730590904571

Rodríguez-Fidalgo, María-Isabel; Ruiz-Paz, Yanira; Paíno-Ambrosio, Adriana (2019). “\#EleccionesMéxico2018: Post election analysis of news media's tweets and citizens' feedback". Communication \& society, v. 32, n. 4, pp. $193-206$.

https://doi.org/10.15581/003.32.4.193-205

Rosenberg, Hans; Syed, Shahbaz; Rezaie, Salim (2020). "The Twitter pandemic: the critical role of Twitter in the dissemination of medical information and misinformation during the Covid-19 pandemic". Canadian journal of emergency medicine, v. 22, n. 4, pp. 418-421.

https://doi.org/10.1017/cem.2020.361 
Rovetta, Alessandro; Bhagavathula, Akshaya-Srikanth (2020). “Global infodemiology of Covid-19: focus on Google Web searches and Instagram hashtags". Journal of medical internet research, v. 22, n. 8, e20673.

https://doi.org/10.2196/20673

Ruiz-del-Olmo, Francisco-Javier; Bustos-Díaz, Javier (2020). “Las imágenes publicadas en Twitter como forma de comunicación política. El caso de las elecciones generales del año 2016 en España”. Revista latina de comunicación social, n. 75, pp. 313-326. https://www.doi.org/10.4185/RLCS-2020-1428

Sáez-Martín, Alejandro; Haro-de-Rosario, Arturo; Caba-Pérez, María-del-Carmen (2015). “Using Twitter for dialogic communication: local government strategies in the European Union". Local government studies, v. 41, n. 3, pp. 421-444. https://doi.org/10.1080/03003930.2014.991866

Schillinger, Dean; Chittamuru, Deepti; Ramírez, A. Susana (2020). “From 'infodemics' to health promotion: a novel framework for the role of social media in public health". American journal of public health, v. 110, n. 9, pp. 1393-1396. https://doi.org/10.2105/AJPH.2020.305746

Segura-Mariño, Adriana-Graciela; Paniagua-Rojano, Francisco-Javier; Fernández-Sande, Manuel (2020). “Metodología para evaluar la comunicación universitaria en Facebook y Twitter". Prisma social, n. 28, pp. 127-144.

https://revistaprismasocial.es/article/view/3357

Solomon, Daniel H.; Bucala, Richard; Kaplan, Mariana J.; Nigrovic, Peter A. (2020). "The 'infodemic' of Covid-19”. Arthritis rheumatology, v. 72, n. 11, e41468.

https://doi.org/10.1002/art.41468

Spence, Patric R.; Lachlan, Kenneth A.; Lin, Xialing; Del-Greco, Maria (2015). "Variability in Twitter content across the stages of a natural disaster: implications for crisis communication". Communication quarterly, v. 63, n. 2, pp. 171-186. https://doi.org/10.1080/01463373.2015.1012219

Suau-Gomila, Guillem; Percastre-Mendizábal, Salvador-José; Palà-Navarro, Gemma; Pont-Sorribes, Carles (2017) "Análisis de la comunicación de emergencias en Twitter. El caso del ébola en España". En: Sierra-Sánchez, Javier; Liberal-Ormaechea, Sheila. Uso y aplicación de las redes sociales en el mundo audiovisual y publicitario. Madrid: McGrawhill Education, pp. 119-130. ISBN: 9788448613570

http://eprints.rclis.org/31269/1/Comunicacion-emergencias-Twitter-ebola-Espana.pdf

Tuñón-Navarro, Jorge; Bouza-García, Luis; Carral, Uxía (2019). Comunicación europea ¿A quién doy like para hablar con Europa? Madrid: Dykinson. ISBN: 9788491489771

Velardi, Paola; Stilo, Giovanni; Tozzi, Alberto E.; Gesualdo, Francesco (2014). "Twitter mining for fine-grained syndromic surveillance". Artificial intelligence in medicine, v. 61, n. 3, pp. 153-163.

https://doi.org/10.1016/j.artmed.2014.01.002

Vos, Sarah C.; Buckner, Marjorie M. (2016). "Social media messages in an emerging health crisis: tweeting bird flu". Journal of health communication, v. 21, n. 3, pp. 301-308.

https://doi.org/10.1080/10810730.2015.1064495

Waisbord, Silvio; Amado, Adriana (2017). “Populist communication by digital means: presidential Twitter in Latin America”. Information, communication \& society, v. 20, n. 9, pp. 1330-1346.

https://doi.org/10.1080/1369118X.2017.1328521

Wigand, Dianne-Lux (2010). "Twitter in government: building relationships, one tweet at a time". In: Seventh international conference on information technology: New generations, pp. 563-567.

https://doi.org/10.1109/ITNG.2010.171

World Health Organization (2020). Risk communication and community engagement readiness and response to coronavirus disease (Covid-19). Interine guidance, 19/03/2020.

https://www.who.int/publications/i/item/risk-communication-and-community-engagement-readiness-and-initialresponse-for-novel-coronaviruses-(-ncov)

Xifra, Jordi (2020). “Comunicación corporativa, relaciones públicas y gestión del riesgo reputacional en tiempos del Covid-19". El profesional de la información, v. 29, n. 2, e290220.

https://doi.org/10.3145/epi.2020.mar.20

Yi, Myongho; Oh, Sam-Gyum; Kim, Sunghun (2013). "Comparison of social media use for the U.S. and the Korean governments". Government information quarterly, v. 30, n. 3, pp. 310-317.

https://doi.org/10.1016/j.giq.2013.01.004

Zeraatkar, Kimia; Ahmadi, Maryam (2018). "Trends of infodemiology studies: a scoping review". Health information and libraries journal, v. 35, n. 2, pp. 91-120.

https://doi.org/10.1111/hir.12216 